\title{
Research on Applications of IoT Technologies in Control System of
}

\section{Smart Home}

\author{
Chang Su${ }^{1, a}$, Chao Yang ${ }^{1, b}$, Lina $\mathrm{Hu}^{1, \mathrm{c}}$ \\ ${ }^{1}$ Heilongjiang University of Technology, Jixi, Heilongjiang, China, 158100 \\ ${ }^{a}$ email, ${ }^{b}$ email, ${ }^{c}$ email Ilvfoxna@126.com
}

Keywords: IoT Technologies, Control System, Smart Home

\begin{abstract}
With the rapid development of digital information technology and network technology, information society not only gradually changes people's lifestyle and work habits, but also challenges the traditional residential. Smart home arises at the historic moment. This paper firstly introduces the concept of smart home and IoT technologies briefly and then explores the applications of IoT technologies in control system of smart home from the software and hard perspectives to provide some references for the relevant researchers.
\end{abstract}

\section{Current Situation of Smart Home}

Smart Home is also called intelligent building, which was proposed by the American Association of residential developers in the early 1980s. Some are called home automation, home networking, electronics, home appliances, digital home appliances. Intelligent Home Furnishing can be defined as a process or system, which brings together the essence of the application of modern electronic technology, communication technology, network technology and automation technology in the application environment in residential Home Furnishing foundation; the Home Furnishing life of the various subsystems together, can automatically control, monitoring and management of electrical equipment, and can provide users with safe, convenient, comfortable and beautiful living environment. Home communication equipment, household appliances and home security devices through the home intelligent system will be a variety of relevant information together, for remote monitoring and management, to maintain the safety of the home environment. Compared with the traditional smart home passive static environment, living environment, has become a one of the advantages of intelligent home furnishing is the wisdom of life helper, make the traditional smart home environment is comfortable, efficient, safety and technology also has the modern life, high quality living environment will certainly rise the user's life quality. Although the development of smart home in China started late, but the pace of development is amazing. With the development of economy, technology innovation and social progress, in the domestic policy under the support of many scientific research institutions have launched research on Intelligent smart home, enterprises of household electrical appliance enterprises, telecom operators and high-tech and some advance has invested and developed intelligent home in their implementation plan, the construction of intelligent Home furnishing will become the future of the intelligent community the development trend of quality management of intelligent smart home design scheme and the key research direction has become popular. 


\section{Common Technologies of IoT}

Internet of things (IoT) is known as the third revolution in information technology after the computer and the Internet. It has been applied in a number of fields, such as the intelligent building, intelligent agriculture, intelligent logistics and urban management, etc.

Technology of RFID. RFID, (Frequency Identification Radio), is a non-contact automatic identification technology. It can automatically identify the target object and obtain relevant data through radio frequency signals. It can work in a variety of harsh environment without manual intervention. RFID technology can identify high-speed moving objects and can identify multiple tags at the same time, fast and convenient operation. RFID is an easy to control, simple and practical, and is particularly suitable for automatic control of the flexibility of application technology. It has the unique superiority of other recognition technology cannot match. It can support read - only mode, and can also support the reading and writing operation mode, which can be carried on a high degree of data integration. One of the most basic RFID systems generally consists of three parts: the tag, RFID reader and the antenna. From the most basic principle, the reader through the space channel to send commands to the RF tag, the tag reader to receive commands to make the necessary response, which can achieve radio frequency identification.

Technology of EPC. EPC is the abbreviation of Electronic Product Code, Chinese translation or electronic products, electronic product code encoding. It is a new technology to improve the management level of logistics supply chain and to reduce the cost, and it is a kind of coding system. It is based on system in order to achieve a single product logo. Product code is the next generation of product identification code. It can be the object of the supply chain (including goods, containers, pallets, position etc.) global unique identifier. EPC has a bar code label, is divided into one dimensional bar code and two-dimensional bar code. One dimensional bar code is composed of a set of parallel lines with different width and different reflectivity, and a blank, which are combined according to certain coding rules and technical standards. In the EPC barcode encoding method in two-dimensional space information stored in horizontal and vertical direction of the bar code, called two-dimensional bar code can directly display the English, Chinese, numbers, symbols and graphics.

Technology of Zigbee. ZigBee technology is a kind of two-way wireless communication technology, because of its short transmission distance, easy operation, low power consumption and cost, in the short distance, low power electronic devices, low rate data transmission is widely used, but also has application cycle or intermittent data and data transmission in the low reaction time in part by; widely used in remote sensing and control, intelligent Home Furnishing, smart cars, smart industry, intelligent agriculture and remote medical fields, through the wireless sensor data acquisition, tracking and control to achieve the goal of regional. ZigBee wireless network technology is a kind of wireless communication technology with low transmission distance, low power consumption and high data transmission rate. Mainly suitable for automatic operation and remote monitoring can be integrated with a variety of equipment. ZigBee is a cheap, low-power short-range wireless networking communications technology, widely used in the field of smart home. With the continuous development and application of information technology, ZigBee technology as a new technology of system integration, many domestic enterprises have been in research and development, ZigBee technology is combined with the set of application software development, network transmission, RF technology and hardware control technology. 


\section{Applications of IoT Technologies in Control System of Smart Home}

Applications in Software of Control System of Smart Home. ZigBee local area network transmission distance, the system through the data conversion platform, realizes signal transmission between ZigBee network and GPRS network, and the system is easy to operate, low power consumption and cost, provides a remote control platform of low data rate data between low power electronic equipment. By analyzing the images displayed by the system, it can be automatically judged when people enter the room or fire. Under normal circumstances of the software design, the parameters of various states and the threshold value of the abnormal situation are not the same. The system is mainly composed of family intelligent control module, image processing unit, ARM central control platform, Zigbee wireless transmission module, GPRS wireless communication module and Internet communication. The family intelligent control module mainly includes intelligent security alarm module, intelligent lighting control module and air conditioning remote control module. Each control module is connected with the ARM central control platform through the Zigbee module, which is composed of a star family intelligent control network. At the same time, central control platform can be connected to a USB camera, to achieve real-time monitoring of the home environment. ARM central control platform through the GPRS and the Internet to achieve the connection with the outside, the user can go anywhere at any time through the Internet or mobile phone to control the smart home.

Home Furnishing networking intelligent remote control system main function is: when the glass is broken, Home Furnishing suffered such illegal invasion of the doors and windows are open, the corresponding sensor will detect the information rapidly through the wireless local area network, sending a signal to capture Home Furnishing controlled by the intelligent control center, information center Home Furnishing data conversion after the SMS, MMS is sent to the user's mobile phone, and the controller processing, so as to realize the family security alarm function. Mobile phone as a remote intelligent home control terminal, combined with single-chip technology, GPRS wireless communication technology and RS232 bus technology to build up the control platform. Home Furnishing related information, issued by the respective wireless terminals, the data acquisition terminal acquisition information through information data, and then through the data center after the conversion to SMS or MMS is sent to the mobile phone. The user can through any intelligent mobile phone, check the information in the home, when the user needs the remote control of household appliances, also can give orders through mobile phone short message, GPRS short message module will be transmitted to the household control center, through the controller processing, into the recognition order, to send a command to the appliance control system through the wireless transmission module, a control the system completes the data operation, to achieve remote control of home appliances. Fire control is the use of temperature humidity detector and smoke detector for real-time monitoring of the Home Furnishing, when installed in the interior of the detector detects the temperature is too high, the smoke concentration is too high, the humidity is too low, the concentration of harmful gas exceed the standard, the system will give an alarm, and alarm information is sent through the ZigBee module to Home Furnishing control center, through the information data terminal controller the data, after the conversion, using GSM module to mobile phone SMS, MMS is sent to the mobile phone users and the associated fire control center.

Applications in Hardware of Control System of Smart Home. The sensor module integrates temperature and humidity values, the use of professional digital module collection technology and humidity sensor technology, and contains a composite sensor of temperature and humidity has been calibrated digital signal output. Superior product quality, high reliability, long term stability, humidity calibration of each DHT11 sensor is very accurate, and the calibration coefficients are 
stored in the OTP memory, storage and processing these calibration coefficients in the detection of sensor signals in real time will be called. DHT11 digital temperature and humidity sensor is composed of a resistance type humidity sensitive element and NTC temperature sensor, and high performance 8 bit microcontroller built, the single wire serial interface 4 pin single pin package, the signal transmission distance to meet the indoor use; the integrated system is simple and quick, convenient connection, fast response, strong anti-interference ability and the price is high, super small volume, low power consumption advantages make it become the best choice of the temperature and humidity sensor. The sensor can convert the collected image signal to analog current signal, and through the analog digital conversion, image processing and transmission are carried out. The control module of the system is used in the relay control circuit. In the boot, $+5 \mathrm{~V}$ power and R3 resistance under the action of the transistor in the conduction state, the relay will pull in the state, at this time, if the user to the microcontroller to send a CLR instruction, a base voltage response of the transistor will drop to about $0 \mathrm{~V}$ at the transistor will turn off state relay power release clips. The relay coil with parallel reverse diode absorption reverse electromotive force, and the corresponding protection drive transistor, the relay driving method is easy to use, simple structure, convenient operation, and low price.

Ethernet is a kind of network technology, which is based on CSMA/CD mechanism. It is an important module in the smart home system, which can realize the remote control of the system, the sharing of resources, the management of the system, and the completion of the system, and so on. However, we use the LC1813 chip itself does not have a network interface, it needs to be expanded. Theoretically speaking, the Ethernet chip DM9000 and Ethernet connector RJ45 crystal is directly connected to complete network interface function, but use in this design is the structure of the DM9000+H1102 network isolation transformer +RJ45 to realize the network interface. The design of the main purpose is to isolate the chip, enhancement of signal and interference when connecting different levels of ability and network port to prevent the impact on the system.

\section{Conclusion}

The goal of the applications of IoT technologies in control system in smart home is an intelligent internet system to facilitate our lives. The technologies of Internet of things can connect a large number of home facilities together, and integrated them into a convenient, advanced and rich control system. People can remotely control home appliances, heating devices, ventilation facilities and so on.

\section{References}

[1] Lyu Xianpeng, Liu Yanlong, Wang Xiangguo, Video Engineering, Vol. 37(2013) No 24, p.43-48

[2] Yu Xinhe, Zhang Haohua, Zhao Xiaoshu, Journal of Shenyang Normal University (Natural Science Edition), Vol. 33(2015) No 2, p.281-284

[3] Li Yu, Wang Weixing, Chen Runze, Vol. 23(2016) No 5, p.71-75

[4] Xin Hailiang, Zhong Peisi, Zhu Shaoqi, Yu Yingjing, Application of Electronic Technique, Vol. 38(2013) No 12, p.79-81+85 